\title{
Classification of shift-symmetric no-scale supergravities
}

\author{
David Ciupke $^{a}$ and Lucila Zárate ${ }^{b}$ \\ ${ }^{a}$ Deutsches Elektronen-Synchrotron DESY, Theory Group, \\ D-22603 Hamburg, Germany \\ ${ }^{b}$ Fachbereich Physik der Universität Hamburg, \\ Luruper Chaussee 149, D-22761 Hamburg, Germany \\ E-mail: david.ciupke@desy.de, lucila.zarate@desy.de
}

Abstract: Models of $4 \mathrm{D} \mathcal{N}=1$ supergravity coupled to chiral multiplets with vanishing or positive scalar potential have been denoted as no-scale. Of particular interest in the context of string theory are models which additionally possess a shift-symmetry. In this case there exists a dual description of chiral models in terms of real linear multiplets. We classify all ungauged shift-symmetric no-scale supergravities in both formulations and verify that they match upon dualization. Additionally, we comment on the realizations within effective supergravities descending from string compactifications.

KEYWORDs: Supergravity Models, Flux compactifications, Supersymmetric Effective Theories

ARXIV EPRINT: 1509.00855 


\section{Contents}

1 Introduction 1

2 Matter-coupled $\mathcal{N}=1$ supergravity in four dimensions 3

2.1 Chiral multiplets 3

2.2 Linear multiplets 4

3 Classification of no-scale supergravities $\quad \mathbf{5}$

3.1 General solution of the real homogeneous Monge-Ampère equation 6

3.2 Classification of no-scale supergravities for linear multiplets 8

4 Explicit classes of solutions and remarks on geometry 9

$\begin{array}{ll}4.1 \text { Homogeneous functions } & 10\end{array}$

$\begin{array}{lll}4.2 & \text { Non-homogeneous examples } & 11\end{array}$

$\begin{array}{ll}4.3 & \text { Remarks about curvature and geometry } \\ \end{array}$

$5 \quad$ No-scale Kähler potentials from string theory $\quad \mathbf{1 5}$

$\begin{array}{lll}5.1 & \text { Type IIA/B orientifold flux-compactifications } & 15\end{array}$

5.2 Type IIB flux-compactifications with O3-planes and $\left(\alpha^{\prime}\right)^{3}$-corrections 17

$\begin{array}{lll}5.3 \text { Discussion } & 17\end{array}$

$\begin{array}{ll}\text { A Equivalence to the homogeneous Monge-Ampère equation } & 19\end{array}$

$\begin{array}{ll}\text { B Duality transformation and matching the solutions } & 19\end{array}$

$\begin{array}{ll}\text { B.1 Duality transformation } & 19\end{array}$

$\begin{array}{lll}\text { B.2 Matching the solutions upon dualization } & 21\end{array}$

\section{Introduction}

No-scale models denote classes of matter-coupled supergravities in four dimensions with positive-/negative-semi-definite or vanishing scalar potentials [1, 2]. Originally these theories were conceived and studied for phenomenological purposes [3]. Later on it was realized that no-scale supergravities arise as the low energy effective actions of certain superstring compactifications [4]. In particular, in compactifications of type II string theory the resulting 4D supergravity also enjoys a perturbative Peccei-Quinn shift-symmetry. Motivated by these examples, it is the purpose of this paper to classify all ungauged shift-symmetric no-scale supergravities. ${ }^{1}$

\footnotetext{
${ }^{1}$ In the following we will only regard ungauged models. A recent example of a gauged no-scale supergravity with shift-symmetry can be found in [5].
} 
For four-dimensional $\mathcal{N}=1$ supergravity coupled to a collection of chiral multiplets $T_{i}$ described via a shift-symmetric Kähler potential $K$ and superpotential $W$, the no-scale condition is defined as

$$
K^{T_{i} \bar{T}_{\bar{\jmath}}} K_{T_{i}} K_{\bar{T}_{\bar{\jmath}}}=p, \quad \text { with } \quad p \in \mathbb{R}
$$

where $K_{T_{i}}=\partial K / \partial T_{i}$ and $K^{T_{i} \bar{T}_{\bar{\jmath}}}$ denotes the inverse Kähler metric. We read this condition as a differential equation for $K$ and, hence, the classification of all shift-symmetric noscale supergravities amounts to solving this differential equation in full generality. As a first step towards this goal, we show that eq. (1.1) is equivalent to the homogeneous Monge-Ampère equation. ${ }^{2}$ More precisely, due to the shift-symmetry one obtains the real version of the homogeneous Monge-Ampère equation, which we then solve with standard methods of partial differential equations. The resulting solution is of a semi-explicit form and generalizes the solution for the special three-field case found in [6]. ${ }^{3}$

Furthermore, supergravity coupled to chiral multiplets enjoying the aforementioned Peccei-Quinn shift-symmetry has a dual description in terms of real linear multiplets $[8,9]$. This dual description has been employed in the context of string compactifications where it often provides a more elegant framework to describe the dynamics of the shift-symmetric scalars, as for instance in [10]. In a theory of linear multiplets the no-scale condition takes a simple form and in this paper we determine its general solution, which in contrast to the chiral case is explicit. Moreover, as a consistency check we demonstrate that the individual solutions to the no-scale condition in the formulation with chiral and linear multiplets match upon dualization.

In the second part of this paper we use the general, semi-explicit solution in the chiral formulation to construct explicit examples. In particular we recover the special class of models where $K$ is given as a logarithm of a homogeneous function. The no-scale property of such Kähler potentials was already pointed out in [11]. However, our general solution shows that Kähler potentials with such a homogeneity property form merely a special subclass among all possible explicit solutions. Thus, it is interesting to determine explicit, non-homogeneous models. For illustrative purposes we construct such solutions both of a more general type and for the special two-field case.

Kähler potentials with homogeneity property are particularly interesting in the context of stringy effective actions. We present an overview of such Kähler potentials descending from various compactifications of string theories. The homogeneity is an exact property of IIA/B orientifold flux-compactifications at tree level and persists even when including string tree-level perturbative $\alpha^{\prime}$-corrections. It can also be found in compactifications of the heterotic string, but is confined there to the dilaton sector and large volume limit respectively. The homogeneity implies the existence of an additional Killing vector of the Kähler manifold related to dilatations. The associated scaling behaviour of the effective Lagrangian is an exact property of string theory at tree level as pointed out in [4]. This

\footnotetext{
${ }^{2}$ An alternative proof of this equivalence was also given in [2].

${ }^{3}$ Note that, an alternative, fully implicit solution to the real homogeneous Monge-Ampère equation was already given in [7].
} 
observation has two interesting consequences, the first being that a scaling symmetry is not a necessary feature of no-scale models. The second is that if stringy no-scale supergravities after inclusion of $g_{s}$-corrections exist, then they will most likely fall into the class of nonhomogeneous functions, whose existence we demonstrated.

This paper is organized as follows. In sections 2.1 and 2.2 we introduce the relevant notation of $4 \mathrm{D} \mathcal{N}=1$ supergravity coupled to chiral and real linear superfields respectively. In section 3.1 we solve the homogeneous real Monge-Ampère equation and, thus, classify shift-symmetric no-scale supergravities for chiral superfields. We derive the corresponding no-scale supergravities with linear superfields in section 3.2. In section 4 we provide explicit examples of the solutions described above and make some remarks about the respective geometries. Finally in section 5 we study the structure of no-scale models descending from string theory in detail. A proof of the equivalence between the Monge-Ampère equation and the no-scale condition can be found in appendix A. In addition, in appendix B we give the duality transformation between chiral and linear theories and explicitly show that the corresponding solutions match via dualization.

\section{Matter-coupled $\mathcal{N}=1$ supergravity in four dimensions}

\subsection{Chiral multiplets}

We begin by reviewing $\mathcal{N}=1$ supergravity coupled to a collection of chiral superfields $T_{i}$ , $i=1, \ldots, n$ in four dimensions. ${ }^{4}$ In the following we partially adopt the notation and conventions of $[9,12]$. (Anti-) chiral superfields are defined by the condition

$$
\overline{\mathcal{D}}^{\dot{\alpha}} T_{i}=\mathcal{D}_{\alpha} \bar{T}_{\bar{\jmath}}=0,
$$

where $\overline{\mathcal{D}}^{\dot{\alpha}}$ and $\mathcal{D}_{\alpha}$ denote the covariant spinorial derivatives. The on-shell bosonic degrees of freedom of the chiral multiplets are complex scalars $T_{i}$, which parametrize a Kähler manifold $\mathcal{M}$ with Kähler potential $K\left(T_{i}, \bar{T}_{\bar{\jmath}}\right)$. The coupling of the chiral superfields to supergravity can be conveniently performed in curved superspace via the Lagrangian

$$
\mathcal{L}=-3 \int E+\frac{1}{2} \int \frac{E}{R} \mathrm{e}^{K / 2} W\left(T_{i}\right)+\frac{1}{2} \int \frac{E}{\bar{R}} \mathrm{e}^{K / 2} \bar{W}\left(\bar{T}_{\bar{\jmath}}\right),
$$

where $W\left(T_{i}\right)$ is the superpotential, $R$ the curvature superfield and $E$ denotes the superdeterminant of the super-vielbein and implicitly depends on $K{ }^{5}$ The corresponding on-shell Lagrangian for the bosonic components reads

$$
e^{-1} \mathcal{L}=-\frac{1}{2} \mathcal{R}-K_{T_{i} \bar{T}_{\bar{\jmath}}} \partial_{\mu} T_{i} \partial^{\mu} \bar{T}_{\bar{\jmath}}-V\left(T_{i}, \bar{T}_{\bar{\jmath}}\right),
$$

where $\mathcal{R}$ denotes the space-time scalar curvature, $e$ the determinant of the vielbein and $K_{T_{i} \bar{T}_{\bar{\jmath}}}=\frac{\partial}{\partial T_{i}} \frac{\partial}{\partial \bar{T}_{\bar{\jmath}}} K$ the Kähler metric. Furthermore the scalar potential is given by

$$
V\left(T_{i}, \bar{T}_{\bar{\jmath}}\right)=\mathrm{e}^{K}\left(K^{T_{i} \bar{T}_{\bar{\jmath}}} D_{T_{i}} W D_{\bar{T}_{\bar{\jmath}}} \bar{W}-3|W|^{2}\right),
$$

\footnotetext{
${ }^{4}$ All the results of this paper directly extrapolate to theories with $\mathcal{N}=2$ supersymmetry in three dimensions.

${ }^{5}$ The integration over the Grassmann variables is implicit in this notation.
} 
where $K^{T_{i} \bar{T}_{\bar{\jmath}}}$ describes the inverse Kähler metric and $D_{T_{i}} W=\frac{\partial}{\partial T_{i}} W+W \frac{\partial}{\partial T_{i}} K$ the Kählercovariant derivative. Defining the function

$$
G\left(T_{i}, \bar{T}_{\bar{\jmath}}\right)=K\left(T_{i}, \bar{T}_{\bar{\jmath}}\right)+\ln \left(\left|W\left(T_{i}\right)\right|^{2}\right),
$$

we can recast the scalar potential into the form

$$
V\left(T_{i}, \bar{T}_{\bar{\jmath}}\right)=\mathrm{e}^{G}\left(G_{T_{i}} G^{T_{i} \bar{T}_{\bar{\jmath}}} G_{\bar{T}_{\bar{\jmath}}}-3\right),
$$

where $G_{T_{i}}=\frac{\partial}{\partial T_{i}} G$ and $G^{T_{i} \bar{T}_{\bar{\jmath}}}$ denotes the inverse of $G_{T_{i} \bar{T}_{\bar{\jmath}}}=\frac{\partial}{\partial T_{i}} \frac{\partial}{\partial \bar{T}_{\bar{\jmath}}} G$. In this paper we are interested in the special class of theories which are of the no-scale type. These are defined via the property

$$
G_{T_{i}} G^{T_{i} \bar{T}_{\bar{\jmath}}} G_{\bar{T}_{\bar{\jmath}}}=p, \quad p \in \mathbb{R} .
$$

From eq. (2.6) it follows that these theories satisfy

$$
V>0 \text { for } p>3, \quad V=0 \text { for } p=3 \text { and } V<0 \text { for } p<3 .
$$

Note that all such models are related via a rescaling of the function $G$. Besides eq. (2.7) one sometimes finds a second notion of no-scale models. This corresponds to a no-scale type condition on the Kähler potential, i.e.

$$
K_{T_{i}} K^{T_{i} \bar{T}_{\bar{\jmath}}} K_{\bar{T}_{\bar{\jmath}}}=p, \quad p \in \mathbb{R} .
$$

This condition in general does not imply positivity or negativity of the scalar potential and is not invariant under Kähler transformations. In the following we will denote models satisfying eq. (2.9) as weakly no-scale. Note also that, if we additionally impose a constant superpotential then eq. (2.9) and eq. (2.7) are equivalent.

\subsection{Linear multiplets}

In this section we will review $\mathcal{N}=1$ supergravity in four dimensions coupled to $n$ linear multiplets $L^{i}$. The latter provide a dual description of chiral models with a Peccei-Quinn shift-symmetry. As in the previous section we adopt the notation and formalism of $[9,12]$. Linear multiplets are defined via the constraint equations

$$
\left(D^{2}-8 \bar{R}\right) L^{i}=\left(\bar{D}^{2}-8 R\right) L^{i}=0 .
$$

The respective bosonic degrees of freedom are given by $\left(L^{i}, B_{2}^{i}\right)$, where $L^{i}$ is a real scalar and $B_{2}^{i}$ a two-form. Contrary to the chiral superfields, the off-shell spectrum of the linear multiplets does not contain any auxiliary field. The geometry of the scalar fields is indirectly determined via two real functions $K\left(L^{i}\right)$ and $F\left(L^{i}\right)$, the former being identical to the Kähler potential in the dual chiral formulation. ${ }^{6}$ We will refer to it as Kähler potential also in theories with linear multiplets. The respective superspace-Lagrangian reads

$$
\mathcal{L}=-3 \int E F\left(L^{i}\right)+\frac{1}{2} \int \frac{E}{R} \mathrm{e}^{K / 2} W+\frac{1}{2} \int \frac{E}{\bar{R}} \mathrm{e}^{K / 2} \bar{W},
$$

\footnotetext{
${ }^{6}$ More precisely, upon dualizing the theory of linear multiplets to a theory with chiral multiplets, as we discuss in detail in appendix B, we identify $K\left(L^{i}(T)\right)$ as the Kähler potential of the chiral theory.
} 
where $W$ is a constant superpotential and $F$ is the aforementioned real function. In the Einstein-frame it is implicitly related to the Kähler potential as follows

$$
1-\frac{1}{3} L^{i} K_{L^{i}}=F-L^{i} F_{L^{i}}
$$

where we abbreviate derivatives with respect to the real scalars as $K_{L^{i}}$ and $F_{L^{i}}$ respectively. The metric inside the kinetic terms is derived from the so-called kinetic or Hessian potential, which is defined as

$$
\tilde{K}\left(L^{i}\right)=K\left(L^{i}\right)-3 F\left(L^{i}\right)
$$

The respective metric $\tilde{K}_{L^{i} L^{j}}$ is understood as a metric on the underlying real manifold, which is parametrized by the scalars $L^{i}$. We are now in the position to write down the bosonic component Lagrangian derived from eq. $(2.11)[9,12]$

$$
e^{-1} \mathcal{L}=-\frac{1}{2} \mathcal{R}+\frac{1}{4} \tilde{K}_{L^{i} L^{j}} \partial_{\mu} L^{i} \partial^{\mu} L^{j}+\frac{1}{4} \tilde{K}_{L^{i} L^{j}} H_{\mu \nu \rho}^{i} H^{\mu \nu \rho j}-V\left(L^{i}\right)
$$

where $H=d B_{2}$ denotes the field strength of the two-form and the scalar potential reads

$$
V\left(L^{i}\right)=\mathrm{e}^{K}\left(L^{i} K_{L^{i}}-3\right)|W|^{2}
$$

In analogy to the previous section we can define no-scale models via the property

$$
L^{i} K_{L^{i}}=p, \quad p \in \mathbb{R} .
$$

We immediately observe that any such theory fulfills eq. (2.8).

The dualization of the theory in eq. (2.14) to a chiral theory is explained in appendix B.1. There we reproduce the important steps of this procedure and show the matching of the scalar potentials explicitly.

\section{Classification of no-scale supergravities}

In eq. (2.7) we introduced the definition of no-scale supergravities for a collection of chiral fields. This definition can be read as a differential equation for the function $G$, which we call no-scale differential equation from now on. Classification of no-scale supergravities, thus, amounts to finding the most general solution to this differential equation and demanding that the resulting theory fulfills all necessary additional requirements. For instance $G$ has to yield a positive-definite Kähler metric. ${ }^{7}$

Solutions to the general no-scale differential equation for $G$ defined in eq. (2.5) are in a one-to-one correspondence with solutions to the homogeneous complex Monge-Ampère equation (HCMA). More precisely the HCMA reads

$$
\operatorname{det}\left(Y_{T_{i} \bar{T}_{\bar{j}}}\right)=0 .
$$

\footnotetext{
${ }^{7}$ Here we only determine the solution to the differential equation. To obtain explicit examples, one needs to check the positivity of the Kähler metric.
} 
The equivalence of the HCMA to the no-scale condition can be stated in the following way: for every solution $Y$ of eq. (3.1) we have a no-scale supergravity with

$$
G=-p \ln Y
$$

and vice versa. This correspondence is demonstrated explicitly in appendix A. Note that an alternative proof of this equivalence was already given in [2].

In the following we concentrate on no-scale supergravities with a Peccei-Quinn shiftsymmetry. In this case the no-scale differential equation is equivalent to the homogeneous real Monge-Ampère equation (HRMA). We derive the general solution to this equation and, thus, provide a classification of the respective no-scale models. A semi-explicit solution to the HRMA for the special case with only three fields (that is $n=3$ ) was given a long time ago in $[6] .^{8}$ It turns out that our result coincides with the solution in [6] for $n=3$. In the second part of this section we determine the general solution to the no-scale condition in eq. (2.16) for a theory with linear multiplets. As a consistency check we explicitly perform the dualization to chiral fields in appendix B and demonstrate that the dual theory matches precisely with the general solution, which we obtained via the HRMA equation.

\subsection{General solution of the real homogeneous Monge-Ampère equation}

Let us now turn to the special case, in which the theory possesses a Peccei-Quinn shiftsymmetry. More explicitly this means that the theory is invariant under the transformations

$$
T_{j}-\bar{T}_{j} \rightarrow T_{j}-\bar{T}_{j}+i c_{j}, \quad j=1, \ldots, n,
$$

where $c_{j} \in \mathbb{R}$. Without loss of generality we can assume that the theory is described via a Kähler potential of the type $K(T+\bar{T})$ and a constant superpotential. ${ }^{9}$ This reduces the no-scale condition to the differential equation for the Kähler potential in eq. (2.9), restricting the corresponding Kähler manifold $\mathcal{M}$. From now on it is convenient to regard $\mathcal{M}$ as a real Riemannian manifold being parametrized by the real and imaginary parts of $T_{i}$. Introducing the notation

$$
\phi_{i}=\frac{1}{2}\left(T_{i}+\bar{T}_{i}\right),
$$

allows to rewrite eq. (3.1) as the HRMA

$$
\operatorname{det}\left(Y_{\phi_{i} \phi_{j}}\right)=0 \text {. }
$$

We now deduce the general solution to the HRMA constructively. First, note that eq. (3.5) is equivalent to solving the eigenvalue equation

$$
Y_{\phi_{i} \phi_{j}}\left(\phi_{1}, \ldots, \phi_{n}\right) v_{j}\left(\phi_{1}, \ldots, \phi_{n}\right)=0, \quad i=1, \ldots, n,
$$

\footnotetext{
${ }^{8}$ The general solution can also be expressed in a different form by means of an analogy with hydrodynamics [7]. However, the structure of this solution is fully implicit. Furthermore, the relation to the result of [6] was investigated in [13]. It is also worth noting that the HRMA can be expressed as the Euler-Lagrange equation for a Lagrangian describing a theory of galileons $[13,14]$.

${ }^{9}$ By performing an appropriate Kähler transformation on a general theory with this shift-symmetry one can always bring the Kähler potential and superpotential to this form.
} 
where $v_{j}\left(\phi_{1}, \ldots, \phi_{n}\right)$ denotes the respective eigenvector. ${ }^{10}$ By defining

$$
Y_{\phi_{i}}\left(\phi_{1}, \ldots, \phi_{n}\right)=Z^{i}\left(\phi_{1}, \ldots, \phi_{n}\right),
$$

we can rewrite eq. (3.6) as

$$
v_{j} \partial_{\phi_{j}} Z^{i}=0 .
$$

Eq. (3.7) and (3.8) constitute a system of first order homogeneous, linear partial differential equations which is equivalent to eq. (3.6). We proceed by solving this system iteratively, that is by integrating eq. (3.8) first. The latter can be understood geometrically in terms of a vector field $\chi$, given in local (real) coordinates as $\chi=v_{j} \partial_{\phi_{j}}$, which annihilates a collection of functions $Z^{1}, \ldots, Z^{n}$. To this vector field we can associate an integral curve

$$
\gamma_{\chi}: I \longrightarrow \mathcal{M}, \quad I \subset \mathbb{R}
$$

such that ${ }^{11}$

$$
\frac{\mathrm{d}}{\mathrm{d} s} \gamma_{\chi}(s)=\chi\left(\gamma_{\chi}(s)\right)
$$

Eq. (3.8) implies that the functions $Z^{i}$ are constant along the integral curve $\gamma_{\chi}$

$$
\frac{\mathrm{d}}{\mathrm{d} s} Z^{i}\left(\gamma_{\chi}(s)\right)=0
$$

We can define a new coordinate system

$$
\left(\phi_{1}, \ldots, \phi_{n}\right) \longrightarrow\left(u_{1}\left(\phi_{i}\right), \ldots, u_{n}\left(\phi_{i}\right)\right), \quad \text { with } \frac{\partial}{\partial u_{n}}=\chi,
$$

that is $u_{n}$ is a coordinate parametrizing $\gamma_{\chi}$ and the remaining coordinates $u_{\alpha}, \alpha=1, \ldots, n-$ 1 are chosen appropriately. ${ }^{12}$ Thus, the solution to eq. (3.11) reads

$$
Z^{i}\left(\phi_{1}, \ldots, \phi_{n}\right)=g^{i}\left(u_{1}\left(\phi_{j}\right), \ldots, u_{n-1}\left(\phi_{j}\right)\right),
$$

where $g^{i}$ is an arbitrary function. It remains to integrate eq. (3.7). Using the fact that we performed a coordinate transformation and eq. (3.13), we can rewrite eq. (3.7) as

$$
\partial_{u_{j}} Y\left(\phi_{1}\left(u_{k}\right), \ldots, \phi_{n}\left(u_{k}\right)\right)=g^{i}\left(u_{1}, \cdots, u_{n-1}\right) \frac{\partial \phi_{i}}{\partial u_{j}}, \quad j=1, \ldots, n .
$$

Specifically we have

$$
\partial_{u_{n}}\left(Y-g^{i} \phi_{i}\right)=0,
$$

which can be integrated directly

$$
Y\left(\phi_{1}, \ldots, \phi_{n}\right)=\phi_{i} g^{i}\left(u_{1}\left(\phi_{k}\right), \ldots, u_{n-1}\left(\phi_{k}\right)\right)+\tilde{Y}\left(u_{1}\left(\phi_{k}\right), \ldots, u_{n-1}\left(\phi_{k}\right)\right) .
$$

\footnotetext{
${ }^{10} \mathrm{In}$ appendix A we show that $v_{j}=K^{T_{j}}$. Here, we introduced a new symbol for the eigenvector for clarity.

${ }^{11}$ Since from appendix A we know that $\chi=K^{T_{i}} \partial_{\phi_{i}}, \chi$ has to be a nowhere vanishing vector field as demanded by the no-scale condition in eq. (2.9). This guarantees that the integral curve exists in the local patch we are regarding.

${ }^{12}$ The imaginary part of $T_{i}$ are unchanged under this coordinate transformation. Note that, this coordinate transformation does not respect the complex structure of the Kähler manifold. However, since the final result is expressed in terms of the original coordinates, the complex structure is restored.
} 
We have to make sure the above integrated form of $Y$ still satisfies eq. (3.7). To this end we compute $Y_{\phi_{i}}$

$$
Y_{\phi_{i}}=g^{i}+\left(\phi_{j} \partial_{u_{\alpha}} g^{j}+\partial_{u_{\alpha}} \tilde{Y}\right) \frac{\partial u_{\alpha}}{\partial \phi_{i}},
$$

and, thus, the second term on the r.h.s. of the above equation has to vanish. This leads to the supplementary constraint equations

$$
\phi_{j} \partial_{u_{\alpha}} g^{j}+\partial_{u_{\alpha}} \tilde{Y}=0, \quad \alpha=1, \ldots, n-1 .
$$

In sum, the solution to the HRMA is given by eq. (3.16) together with the constraints in eq. (3.18). Since it is necessary to solve the additional constraint equations, the solution is of a semi-explicit form. For later purposes we rewrite eq. (3.16) as

$$
Y\left(\phi_{1}, \ldots, \phi_{n}\right)=\phi_{i} Y_{\phi_{i}}\left(\phi_{1}, \ldots, \phi_{n}\right)+\tilde{Y}\left(u_{1}\left(\phi_{k}\right), \ldots, u_{n-1}\left(\phi_{k}\right)\right) .
$$

As a final remark, it is worth noting that eq. (3.5) is invariant under the following transformations

$$
\begin{aligned}
& Y\left(\phi_{i}\right) \longrightarrow \lambda Y\left(\varphi_{i}\right)+b^{i} \phi_{i}+c, \quad b^{i}, c, \lambda \in \mathbb{R}, \text { where } \\
& \varphi_{i}\left(\phi_{j}\right)=A_{i}^{j} \phi_{j}+a_{i}, \quad A \in G L(n), a_{i} \in \mathbb{R} .
\end{aligned}
$$

Once a particular solution to the HRMA is identified, one can use the symmetrytransformations to obtain additional solutions. In this sense solutions form equivalence classes under the transformation rules. In particular, we will later on use this to identify seemingly distinct solutions.

\subsection{Classification of no-scale supergravities for linear multiplets}

We now turn to the formulation of supergravity coupled to linear multiplets, which we reviewed in section 2.2. Recall the definition of a no-scale supergravity in the linear multiplet formalism, which reads

$$
L^{i} K_{L^{i}}=p, \quad p \in \mathbb{R} .
$$

We read this equation as a differential equation for the Kähler potential $K$. Contrary to the no-scale differential equation for the chiral multiplets eq. (3.21) is of first order. To obtain the correct number of degrees of freedom we need another first order differential equation. The missing equation is the Einstein-frame normalization condition in eq. (2.12), which relates the function $F$ to the Kähler potential. More precisely, inserting eq. (3.21) into the Einstein-frame condition we obtain

$$
F-L^{i} F_{L^{i}}=1-\frac{p}{3}
$$

Eqs. (3.21) and (3.22) describe a system of first order differential equations, which specifies no-scale supergravities in the linear multiplet formalism and we now solve this system explicitly. In the spirit of eq. (3.2) we introduce

$$
K\left(L^{i}\right)=-p \ln \left(Y\left(L^{i}\right)\right)
$$


which when inserted in eq. (3.21) yields

$$
L^{i} Y_{L^{i}}=-Y .
$$

Note that a homogeneous function $\mathcal{F}\left(\phi_{1}, \ldots, \phi_{n}\right)$ of degree $m$ is defined via the property

$$
\mathcal{F}\left(\lambda \phi_{1}, \ldots, \lambda \phi_{n}\right)=\lambda^{m} \mathcal{F}\left(\phi_{1}, \ldots, \phi_{n}\right), \quad \forall \lambda \in \mathbb{R} .
$$

Alternatively homogeneous functions can be defined as the general solution to the following differential equation

$$
m \mathcal{F}=\phi_{i} \mathcal{F}_{\phi_{i}} .
$$

Thus, the general solution to eq. (3.24) is a homogeneous function of degree -1 . In particular eq. (3.25) implies that $Y$ satisfies ${ }^{13}$

$$
Y\left(L^{1}, \ldots, L^{n}\right)=\frac{1}{L^{1}} Y\left(\frac{L^{2}}{L^{1}}, \ldots, \frac{L^{n}}{L^{1}}\right) .
$$

It remains to solve eq. (3.22), which is an inhomogeneous linear differential equation. The general solution can be written as the sum of the general solution to the respective homogeneous equation and a particular solution to the inhomogeneous equation. The corresponding homogeneous equation is the differential equation for a homogeneous function of degree one, so that we can write the solution as

$$
F\left(L^{i}\right)=F_{(1)}\left(L^{i}\right)+1-\frac{p}{3},
$$

where $F_{(1)}$ is homogeneous of degree one. As promised we find that the solution to the system of eqs. (3.21) and (3.22) can be displayed explicitly.

\section{Explicit classes of solutions and remarks on geometry}

In the previous section we derived the general solution to the no-scale condition both for a theory of chiral multiplets with a shift-symmetry as well as for a theory with linear multiplets. Furthermore, in appendix B we show that these solutions match upon dualization. The result for the chiral theory, which is displayed in eq. (3.16) and (3.18), is semi-explicit. To find fully explicit solutions we need to impose additional conditions. It will be the purpose of this section to construct classes of explicit solutions and make a comparison to those examples, which are already known in the literature. In the second part of this section we will derive certain geometric statements, which on one hand illustrate the geometric meaning of the no-scale condition, and on the other hand help to understand the difference between the classes of solutions which we present in the first part of this section.

Before turning to the examples, let us make a few comments on how to interpret the solution in eq. (3.16) and (3.18). One may regard eqs. (3.18) as a system of differential equations, which needs to be solved in order to find explicit solutions. The input data for the initial value problem corresponding to eq. (3.5) are two functions, that are allowed

\footnotetext{
${ }^{13}$ Strictly speaking the identification is given as $Y\left(L^{1}, \ldots, L^{n}\right)=\frac{1}{L^{1}} Y\left(1, \frac{L^{2}}{L^{1}}, \ldots, \frac{L^{n}}{L^{1}}\right)$.
} 
to depend on $(n-1)$ variables each. The standard procedure (although complicated) is to choose two functions out of the collection $\left(g^{1}, \ldots, g^{n}, \tilde{Y}\right)$ and determine the remaining functions by solving the system in eqs. (3.18). In this line of reasoning the functions $u_{\alpha}(\phi)$ are related to the coordinates with respect to which we define a hyper-surface, on which we specify the initial data. For instance we could choose the initial data on a hyper surface defined via the equation $u_{n}=0$. For the practical purpose of finding explicit solutions it turns out to be advantageous to simply choose all $\left(g^{1}, \ldots, g^{n}, \tilde{Y}\right)$ and interpret eqs. (3.18) algebraically, that is to solve for $u_{\alpha}$. As long as the system is solvable this procedure automatically yields a solution. As we will see it is in fact not always necessary to choose all $\left(g^{1}, \ldots, g^{n}, \tilde{Y}\right)$, but possibly only a subclass.

\subsection{Homogeneous functions}

We now apply the aforementioned procedure to construct a class of explicit solutions for the chiral theory. Let us choose

$$
\tilde{Y}=0
$$

while keeping $g^{1}, \ldots, g^{n}$ arbitrary. Eq. (3.19) then reduces to

$$
Y\left(\phi_{1}, \ldots, \phi_{n}\right)=\phi_{i} Y_{\phi_{i}}\left(\phi_{1}, \ldots, \phi_{n}\right) .
$$

In eq. (4.2) we recognize the differential equation, which defines a homogeneous function of degree one. ${ }^{14}$ Let us also display the form of the solutions satisfying eq. (4.1) in the dual linear multiplet formulation, see appendix B for the relations between the solutions in both formalisms. Using eqs. (4.1), (B.14) and (3.28) we obtain that the homogeneous solutions correspond to a constant $F$ given by

$$
F=1-\frac{p}{3}
$$

Furthermore, recall that the HRMA is equivalent to the existence of an eigenvector with eigenvalue zero. In appendix A we determine this eigenvector explicitly. For the class of homogeneous functions it turns out that the components of this eigenvector are particularly simple. Namely, taking the derivative of eq. (4.2) yields the looked-for eigenvalue equation (3.6) with corresponding eigenvector

$$
v_{j}=\phi_{j}
$$

Similarly direct computation yields

$$
K^{T_{i}}=-2 \phi_{i}
$$

which is in agreement with the identification of the eigenvector according to appendix A.

Logarithmic Kähler potentials which satisfy eq. (4.2) are important in the context of moduli spaces of superstring compactifications. A well known example is the large-volume

\footnotetext{
${ }^{14}$ Recall that solutions form equivalence classes, where individual representatives are related by the symmetries given in eq. (3.20). In particular a homogeneous function in general transforms into an inhomogeneous one.
} 
limit of the Kähler potentials of the moduli spaces of type IIB Calabi-Yau orientifold compactifications with $03 / O 7$-planes $[10,15,16]$. Here the Kähler moduli sector has a perturbative Peccei-Quinn shift symmetry and, thus, the superpotential is constant along the Kähler moduli directions. The respective Kähler potential here is of the form

$$
K(T, \bar{T})=-2 \ln \mathcal{V}(T, \bar{T}), \quad \mathcal{V}(T, \bar{T})=\mathcal{K}_{i j k} t^{i} t^{j} t^{k}
$$

where $\mathcal{K}_{i j k}$ are the triple intersection numbers and $t^{i}$ are two-cycle volumes. The latter are related to the Kähler moduli through $\operatorname{Re} T_{i}=\frac{3}{2} \mathcal{K}_{i j k} t^{j} t^{k}$. From (4.6) we learn that $\mathcal{V}$ is homogeneous of degree 3 in $t^{i}$ which, in turn implies it is of degree $3 / 2$ in $\operatorname{Re} T_{i}{ }^{15}$ Automatically this leads to the no-scale property

$$
G^{T_{i} \bar{T}_{j}} G_{T_{i}} G_{\bar{T}_{j}}=3 .
$$

Moreover, let us display the Kähler potential in terms of linear multiplets. Using eq. (4.3) we find that $F=0$, since $p=3$ by means of eq. (4.7). Using eq. (B.3) we determine $L^{i}$ to be

$$
L^{i}=\frac{3}{2} \frac{t^{i}}{\mathcal{V}}
$$

We are now in a position to express the Kähler potential as a function of the real linear scalars

$$
K(L)=\ln \left(\frac{8}{27} \mathcal{K}_{i j k} L^{i} L^{j} L^{k}\right)
$$

which as expected is a homogeneous function of degree $3 .{ }^{16}$ We will return to the discussion of moduli spaces of superstring compactifications in section 5, where we will make a broader connection between logarithmic Kähler potentials of a homogeneous type and the geometry of moduli spaces.

\subsection{Non-homogeneous examples}

In the previous section we have displayed an explicit class of solutions to eq. (3.5), namely the homogeneous functions and furthermore hinted on their importance in the context of compactifications of string theory. In this section we want to illustrate explicitly that the general solution in eq. (3.16) encompasses more than just homogeneous functions. Even though these non-homogeneous solutions might look involved they provide new insights into the expected Kähler potentials.

We begin by making a general observation. Given a particular solution $Y_{0}\left(\phi_{1}, \ldots, \phi_{n}\right)$ to the HRMA with $n$ variables $\phi_{1}, \ldots, \phi_{n}$ one can construct a solution $Y$ to the HRMA with $n+m$ variables $M_{I}=\left(\phi_{i}, \rho_{a}\right)$, where $i=1, \ldots, n$ and $a=1, \ldots, m$ via

$$
Y\left(M_{I}\right)=Y_{0}\left(\phi_{1}+Q_{1}\left(\rho_{a}\right), \ldots, \phi_{n}+Q_{n}\left(\rho_{a}\right)\right),
$$

\footnotetext{
${ }^{15}$ Notice that here $Y=\mathcal{V}^{\frac{2}{3}}$ and, thus, $Y$ is a homogeneous function of degree one.

${ }^{16}$ Analogously to the previous footnote, in this example $Y(L)=\left(\frac{8}{27} \mathcal{K}_{i j k} L^{i} L^{j} L^{k}\right)^{-2 / 3}$ and, hence, $Y(L)$ is an homogeneous function of degree -1 .
} 
with $Q_{1}, \ldots, Q_{n}$ being a collection of real-valued functions. Direct computation yields that $Y$ in eq. (4.10) obeys

$$
Y_{M_{I} M_{J}} v_{J}=0,
$$

where $v_{J}=\left(v_{i}^{(0)}, 0\right)$ and $v^{(0)}$ denotes the eigenvector to eigenvalue zero associated with the fact that $Y_{0}$ solves the HRMA. For the simple case $n=1$ the above construction leads to a solution of the form

$$
Y=\phi_{1}+Q_{1}\left(\rho_{1}, \ldots, \rho_{m}\right),
$$

which for general $Q_{1}$ is non-homogeneous. Note that, the linear term in the above equation can also be absorbed by performing a symmetry-transformation of the type in eq. (3.20). Thereby the function transforms into another function which depends only on $\rho_{1}, \ldots, \rho_{m}$ and, thus, trivially solves the HRMA. Furthermore, one may consider $Y_{0}$ to be a homogeneous function of degree one, which for general $Q_{i}$ again leads to non-homogeneous examples. However, note that solutions of the type in eq. (4.10) are very special, since they effectively only depend on $n$ variables.

Thus, it is interesting to construct solutions which fully exemplify the generality of eq. (3.13). To this end we need to solve the constraint equations in eq. (3.18) which in general is a delicate task. In what follows we construct solutions to eq. (3.18) for the special case of two fields, that is $n=2$. The respective Kähler potentials were so far unknown in the literature.

For a two-dimensional Kähler manifold there is only one coordinate $u$ and one constraint equation. The latter can be recast into the form

$$
\phi_{1}+\phi_{2} \frac{g^{2 \prime}}{g^{1 \prime}}+\frac{\tilde{Y}^{\prime}}{g^{1 \prime}}=0,
$$

provided $g^{1 \prime} \neq 0$ and where $g^{1}, g^{2}$ and $\tilde{Y}$ are functions of $u$. We define $f \equiv g^{2 \prime} / g^{1 \prime}$ and assume $\tilde{Y}^{\prime} / g^{1 \prime}=f^{q}$ with $q \in \mathbb{Z}$, while keeping $g^{1}$ and $f$ arbitrary. Eq. (4.13) can be analytically solved in very few cases. Namely for $q=1,2,3,4$ the roots of eq. (4.13) and, thus, $u$ can be determined explicitly. For $q=1$ the solution can be transformed into a homogeneous function by means of eq. (3.20). For $q=2$, there are two solutions for $u$ given by ${ }^{17}$

$$
u_{ \pm}\left(\phi_{1}, \phi_{2}\right)=f^{-1}\left(-\frac{1}{2} \phi_{2} \pm \sqrt{\frac{1}{4} \phi_{2}^{2}-\phi_{1}}\right) \text {. }
$$

For $q=3,4$ the solutions are quite complicated and we will not display them here. In general expressing $g^{2}$ and $\tilde{Y}$ in terms of $g^{1}$ and $f$, the solution reads

$$
\begin{aligned}
Y\left(\phi_{1}, \phi_{2}\right)= & {\left[\phi_{1}+\phi_{2} f(u)+f^{q}(u)\right] g^{1}(u) } \\
& -\phi_{2} \int \mathrm{d} u g^{1}(u) f^{\prime}(u)-\int \mathrm{d} u g^{1}(u)\left(f^{q}\right)^{\prime}(u),
\end{aligned}
$$

where $u$ is understood as being replaced by the solution $u\left(\phi_{1}, \phi_{2}\right)$ to eq. (4.13). Let us now look for simple particular examples. Setting $q=2$ and $g^{1}(u)=f(u)=u$ the solution reduces to

$$
Y\left(\phi_{1}, \phi_{2}\right)=\frac{1}{12}\left[\phi_{2}^{3}-6 \phi_{1} \phi_{2}+\left(\phi_{2}^{2}-4 \phi_{1}\right)^{3 / 2}\right] .
$$

\footnotetext{
${ }^{17}$ To ensure that $u$ is a real function we have to restrict the field range to the region $\phi_{2}^{2} \geq 4 \phi_{1}$.
} 
Another interesting example is $q=2$ and $g^{1}(u)=u, f(u)=\ln u$, where ${ }^{18}$

$$
Y\left(\phi_{1}, \phi_{2}\right)=\exp \left[-\frac{1}{2}\left(\phi_{2}-\sqrt{\phi_{2}^{2}-4 \phi_{1}}\right)\right]\left(2-\sqrt{\phi_{2}^{2}-4 \phi_{1}}\right) .
$$

Notice here that after replacing $Y$ in the Kähler potential, as defined in eq. (3.2), the first factor drops out of the logarithm. It is somewhat surprising that a Kähler potential of this form obeys the no-scale condition. ${ }^{19}$

\subsection{Remarks about curvature and geometry}

After derivation of the general solution to the no-scale condition for models with shiftsymmetry it is interesting to make statements about the resulting geometries. We will not attempt to compute the components of the Riemann-tensor here, but try to offer some general insights. Firstly, we will derive a statement about the holomorphic sectional curvature for general no-scale models. Secondly, we will derive a geometric distinction between homogeneous and non-homogeneous solutions.

In [17] it was shown that no-scale models have constant holomorphic sectional curvature along a special direction. Let us here slightly generalize the derivation of [17] starting from the general no-scale condition in eq. (2.7). In the following we denote the covariant derivative on the Kähler manifold by $\nabla_{T_{i}}$. This covariant derivative is defined with respect to a metric compatible and hermitian connection. To begin with, taking the covariant derivative of eq. (2.7) we deduce

$$
G_{T_{i}}+G^{T_{k}} \nabla_{T_{i}} G_{T_{k}}=0
$$

where we used the fact that $\nabla_{T_{i}} G_{\bar{T}_{\bar{k}}}=G_{T_{i} \bar{T}_{\bar{k}}}$ as well as metric compatibility. Taking an anti-holomorphic covariant derivative of eq. (4.18) one infers

$$
G_{T_{i} \bar{T}_{\bar{\jmath}}}+\nabla_{T_{i}} G_{T_{k}} \nabla_{\bar{T}_{\bar{\jmath}}} G^{T_{k}}-R_{T_{i} \bar{T}_{\bar{\jmath}} T_{k} \bar{T}_{\bar{l}}} G^{T_{k}} G^{\bar{T}_{\bar{l}}}=0
$$

where $R$ denotes the Riemann tensor. Instead taking a holomorphic derivative of eq. (4.18) yields

$$
2 \nabla_{T_{i}} G_{T_{j}}+G_{T_{k}} \nabla_{T_{i}} \nabla_{T_{j}} G^{T_{k}}=0 .
$$

Using eq. (4.18) and eq. (4.20) we can derive the auxiliary property

$$
\nabla_{T_{i}} G_{T_{k}} \nabla_{\bar{T}_{\bar{j}}} G^{T_{k}}=\nabla_{T_{k}} G_{T_{i}} \nabla_{\bar{T}_{\bar{j}}} G^{T_{k}} .
$$

Altogether and using the no-scale condition in eq. (2.7) we find that

$$
R_{T_{i} \bar{T}_{\bar{\jmath}} T_{k} \bar{T}_{\bar{l}}} G^{T_{i}} G^{\bar{T}_{\bar{\jmath}}} G^{T_{k}} G^{\bar{T}_{\bar{l}}}=2 p,
$$

\footnotetext{
${ }^{18}$ We chose both for eq. (4.16) as well as for $Y$ below the solution $u_{-}$, for which we explicitly checked that the respective $Y$ yield a real valued Kähler potential and a positive-definite Kähler metric in a certain region of field space.

${ }^{19}$ Note also, that by means of a Kähler transformation we can recast the theory in eq. (4.17) as a theory with $W=\exp \left(\frac{p}{4} T_{2}\right)$ and $K=-p \ln (2-\Delta)-\frac{p}{2} \Delta$ where $\Delta\left(\phi_{1}, \phi_{2}\right)=\sqrt{\phi_{2}^{2}-4 \phi_{1}}$.
} 
which states that all no-scale models have constant holomorphic sectional curvature along the vector field $G^{T_{i}}$. Recall from appendix A that this vector field is coincident with the eigenvector (field) to the eigenvalue zero ensuring the validity of the HCMA. More specifically in the shift-symmetric case this tells us that the curvature along the integral curve $\gamma_{D}$ as defined in eq. (3.10) is constant.

Let us now turn to shift-symmetric no-scale models and try to make a geometric distinction between Kähler potentials of homogeneous and non-homogeneous type. Firstly, for the former it turns out that also the Ricci curvature is constant along $G^{T_{k}}$. More precisely contracting eq. (4.19) with an inverse metric and using eq. (4.5) we find

$$
\operatorname{Ric}_{T_{i} \bar{T}_{\bar{\jmath}}} G^{T_{i}} G^{\bar{T}_{\bar{\jmath}}}=2 n,
$$

where Ric denotes the Ricci tensor. ${ }^{20}$ For non-homogeneous solutions one finds that the l.h.s. of eq. (4.23) is in general non-constant. We checked this explicitly for the examples discussed in section 4.2 .

Moreover, homogeneous-type Kähler potentials have an additional isometry. The respective transformations are dilatations, whose infinitesimal version reads

$$
\delta T_{i}=\epsilon T_{i}, \quad \delta \bar{T}_{\bar{\jmath}}=\epsilon \bar{T}_{\bar{\jmath}}
$$

We identify the respective Killing vector as

$$
\Xi=\Xi^{T_{i}} \frac{\partial}{\partial T_{i}}+\Xi^{\bar{T}_{\bar{\jmath}}} \frac{\partial}{\partial \bar{T}_{\bar{\jmath}}}, \quad \text { where } \quad \Xi^{T_{i}}=T_{i}, \quad \Xi^{\bar{T}_{\bar{\jmath}}}=\bar{T}_{\bar{\jmath}} .
$$

Indeed, by direct computation (and using eq. (4.5)) we find

$$
\begin{aligned}
& \nabla_{T_{i}} \Xi_{T_{j}}+\nabla_{T_{j}} \Xi_{T_{i}}=0, \\
& \nabla_{T_{i}} \Xi_{\bar{T}_{\bar{\jmath}}}+\nabla_{\bar{T}_{\bar{\jmath}}} \Xi_{T_{i}}=0,
\end{aligned}
$$

which are the Killing vector equations.

We can promote this isometry to a symmetry of the bosonic sector of the respective supergravity in eq. (2.3) if $V=0$, that is if $p=3$, and if the spacetime-metric does not transform under dilatations. For $p$ arbitrary another interesting situation occurs when the spacetime-metric transforms under dilatations with weight $p$. The respective infinitesimal version reads

$$
\delta g_{\mu \nu}=\epsilon p g_{\mu \nu} .
$$

In this case also the bosonic Lagrangian transforms under the dilatations as

$$
\delta \mathcal{L}=\epsilon p \mathcal{L}
$$

In the context of effective supergravities of string compactifications the scaling property of the Lagrangian plays a role and we will come back to it in the next section.

\footnotetext{
${ }^{20}$ This was also found in [11].
} 


\section{$5 \quad$ No-scale Kähler potentials from string theory}

In the previous section we presented an explicit example of the general solution to the no-scale condition in section (3) both for chiral and linear multiplets, namely type IIB orientifold flux-compactifications with $O 3$-planes. In this section we will study the structure of no-scale models descending from string-theory compactifications in more detail. In general, string-derived no-scale models exhibit a shift-symmetry only in a subsector of the theory and, thus, our setup applies to those cases, in which the total Kähler manifold $\mathcal{M}$ of the theory admits a product structure

$$
\mathcal{M}=\mathcal{M}_{n s} \times \mathcal{M}_{r}
$$

where $\mathcal{M}_{n s}$ is the subspace of scalars with a shift-symmetry obeying the no-scale property and $\mathcal{M}_{r}$ is parametrized by the remaining fields. To guarantee the no-scale property in eq. (2.7) it is also necessary that the superpotential depends only on the fields inside $\mathcal{M}_{r}$. However, in the context of geometric string compactifications this structure is rather special and appears essentially only in an asymptotic regime of the manifold or for special configurations of the compactification data. ${ }^{21} \mathrm{~A}$ particular example is the large volume limit of the heterotic string compactified on a Calabi-Yau threefold [18-20]. In this example the Kähler potential at lowest order in the matter fields is given by a logarithm of a homogeneous function as already pointed out in $[11] .{ }^{22}$

It is interesting to turn to the more general situation, in which the theory has a shift-symmetric Kähler potential but not necessarily a shift-symmetric superpotential. This allows to discuss the weak-type no-scale condition in eq. (2.9). In the following we revisit orientifold compactifications of type IIA/B string theory. These yield 4D theories with a shift-symmetric Kähler potential obeying a weak-type no-scale condition at tree level. ${ }^{23}$ Moreover, this condition holds on the entire scalar manifold and not only at special points, such as e.g. at large volume. As it turns out the respective Kähler potentials are again given by logarithms of homogeneous functions. Furthermore, we will present an example where both the shift-symmetry as well as the weak-type no-scale condition survive perturbative $\alpha^{\prime}$-corrections.

\subsection{Type IIA/B orientifold flux-compactifications}

The low-energy effective supergravities describing type IIB orientifold-compactifications with fluxes and $03 / O 7$-planes or $O 5 / O 9$-planes were determined in [10, 22]. The respective Kähler moduli/dilaton subsectors of the effective actions enjoy a shift-symmetric Kähler potential, which is given as a logarithm of a homogeneous function of degree -4 as stated

\footnotetext{
${ }^{21}$ It is for instance possible that special flux choices in type II orientifold compactifications imply a shiftsymmetry in the superpotential. Furthermore, eq. (5.1) holds in type IIB orientifold flux-compactifications with $O 3$-planes but no $O 7$-planes.

${ }^{22}$ Note furthermore that terms quadratic in the matter fields also have a homogeneity property as pointed out in [11].

${ }^{23}$ Also in Calabi-Yau fourfold-compactifications of M-theory with flux one can find weak-type no-scale conditions where the respective Kähler potential has a homogeneity property [21].
} 
in [12]. ${ }^{24}$ For illustrative purposes we now revisit the $03 / O 7$-plane case and demonstrate the homogeneity explicitly. The relevant Kähler variables here are given by $T_{i}, \tau, G_{a}$, where $\alpha=1, \ldots, h_{+}^{1,1}$ and $a=1, \ldots, h_{-}^{1,1}$ and $h_{ \pm}^{1,1}$ denote the dimension of the even/odd spaces of harmonic $(1,1)$-forms on the Calabi-Yau orientifold. The Kähler potential reads [10]

$$
K(\tau, \bar{\tau}, T, \bar{T}, G, \bar{G})=-\ln (-i(\tau-\bar{\tau}))-2 \ln \left(\frac{1}{6} \mathcal{K}_{i j k} t^{i} t^{j} t^{k}\right)
$$

where $t^{i}$ denote two-cycle volumes as in eq. (4.6) and are understood as being replaced by the solution to the equation

$$
T_{i}+\bar{T}_{i}=\frac{3}{2} \mathcal{K}_{i j k} t^{j} t^{k}+\frac{3 i}{4} \frac{1}{\tau-\bar{\tau}} \mathcal{K}_{i b c}\left(G_{b}-\bar{G}_{b}\right)\left(G_{c}-\bar{G}_{c}\right)
$$

and, thus, implicitly depend on $M_{I}=\left(T_{i}, \tau, G_{a}\right)$. Here, the objects $\mathcal{K}_{i j k}$ and $\mathcal{K}_{i b c}$ denote triple intersection numbers of the compactification manifold. Immediately we observe that $t^{i}$ and, hence, also the Kähler potential has a shift-symmetry with respect to $\operatorname{Im}\left(T_{i}\right), \operatorname{Re}(\tau)$ and $\operatorname{Re}\left(G_{a}\right)$. Moreover, eq. (5.3) implies that $t^{i}$ is a homogeneous function of degree $1 / 2$ in the variables $M_{I}$. Thus, the Kähler potential is given as the logarithm of a homogeneous function of degree -4 . This in turn yields the following no-scale property

$$
K_{M_{I}} K^{M_{I} \bar{M}_{J}} K_{\bar{M}_{J}}=4 .
$$

Note that we can also understand eq. (5.3) as a particular realization of the construction in eq. (4.10) by identifying $\rho_{a}=-i\left(G_{a}-\bar{G}_{a}\right) / 2$ and $Y_{0}$ via eq. (4.6). ${ }^{25}$

Let us remark that since here the superpotential depends on the dilaton [10], the weak no-scale condition alone does not imply positivity of the scalar potential. Nevertheless, it turns out that the scalar potential is positive-semi-definite for eq. (5.2). This can be well understood in a dual description where $T_{i}$ are dualized into linear multiplets [10]. ${ }^{26}$ The effective action of type IIA orientifold flux-compactifications with O6-planes was computed in [22]. Here, the Kähler potential has a shift-symmetry in the real (or imaginary) parts of complex structure, dilaton and Kähler moduli and, furthermore, is given as a logarithm of a homogeneous function of degree -7 . Thus, one has a weak no-scale property

$$
K_{\mathcal{E}_{A}} K^{\mathcal{E}_{A} \overline{\mathcal{E}}_{\bar{B}}} K_{\overline{\mathcal{E}}_{\bar{B}}}=7
$$

where $\mathcal{E}_{A}$ collectively denote all complex scalar fields in the spectrum.

\footnotetext{
${ }^{24}$ This structure appears also in generalized orientifold compactifications, for an overview see for instance [23-26].

${ }^{25}$ In particular this construction can be used to infer that $K_{m_{I}} K^{m_{I} \bar{m}_{J}} K_{\bar{m}_{J}}=3$ where $m_{I}=\left(T_{i}, G_{a}\right)$, that is by setting the dilaton to a constant.

${ }^{26}$ Recall from our discussion in the preceding sections, that for models with a Peccei-Quinn shift-symmetry the no-scale conditions in the chiral theory and in the dual theory with linear multiplets are equivalent to each other. We would like to emphasize here, that this equivalence in general does not hold any longer, if the theory includes additional chiral fields, which do not exhibit a Peccei-Quinn shift-symmetry and hence can not be dualized to linear multiplets.
} 


\subsection{Type IIB flux-compactifications with $O 3$-planes and $\left(\alpha^{\prime}\right)^{3}$-corrections}

The 10D effective action of type IIB string theory receives perturbative $\alpha^{\prime}$ - and $g_{s^{-}}$ corrections. In particular this includes a term at order $\left(\alpha^{\prime}\right)^{3}$ consisting of contractions of four Riemann-tensors [27]. This term can be understood as a four-point scattering amplitude, receiving contributions at string tree-level as well as at string-loop level. For CalabiYau orientifold flux-compactifications with $O 3$-planes a correction to the Kähler potential of the Kähler moduli is induced by this 10D correction and was determined in [16]. At string tree-level the $\alpha^{\prime}$-corrected Kähler potential for the dilaton/Kähler-moduli sector reads

$$
K\left(\tau, \bar{\tau}, T_{i}, \bar{T}_{\bar{\jmath}}\right)=-\ln (-i(\tau-\bar{\tau}))-2 \ln \left(\mathcal{V}\left(T_{i}, \bar{T}_{\bar{\jmath}}\right)+\xi\left[-\frac{i}{2}(\tau-\bar{\tau})\right]^{3 / 2}\right) .
$$

Here $\xi$ is a numerical constant and the Kähler variables $T_{i}$ are related to the volume $\mathcal{V}$ in the same way as in eq. (4.6). Again we have a shift-symmetry both for $T_{i}$ as well as for the dilaton. Moreover, from section 4.1 we know that $\mathcal{V}$ is a homogeneous function of degree $3 / 2$ in $T_{i}+\bar{T}_{i}$. Hence, taking into account the dilaton-dependence we find that $K$ in eq. (5.6) is given by a logarithm of a homogeneous function of degree -4 . This yields the following no-scale property

$$
K_{N_{A}} K^{N_{A} \bar{N}_{B}} K_{\bar{N}_{B}}=4,
$$

where $N_{A}=\left(T_{i}, \tau\right) .{ }^{27}$ To our knowledge eq. (5.7) was so far unknown. Let us emphasize again that this is a weak-type no-scale property. Contrary to the previous example, here the scalar potential is not positive semi-definite [16]. Moreover, it is interesting to note that eq. (5.7) holds no longer after including string-loop corrections to the 10D higher-curvature invariant. It was found in [27] that these corrections essentially coincide with the tree-level $R^{4}$-term, the only difference being that they are suppressed with an additional factor of $g_{s}^{2}$. In turn, we find that the Kähler potential for the effective $4 \mathrm{D}$ action of the Kähler moduli-dilaton subsector after including the one-loop corrections is of the form

$$
\begin{aligned}
K\left(\tau, \bar{\tau}, T_{i}, \bar{T}_{\bar{\jmath}}\right)= & -\ln (-i(\tau-\bar{\tau})) \\
& -2 \ln \left(\mathcal{V}+\xi\left[-\frac{i}{2}(\tau-\bar{\tau})\right]^{3 / 2}+\tilde{\xi}\left[-\frac{i}{2}(\tau-\bar{\tau})\right]^{-1 / 2}\right),
\end{aligned}
$$

where $\tilde{\xi}$ is another numerical factor. We immediately observe that the homogeneity of the Kähler potential is spoiled.

\subsection{Discussion}

One might wonder whether it is possible to understand the form of the Kähler potentials of type II compactifications with orientifolds and in particular the weak no-scale property

\footnotetext{
${ }^{27}$ There is also a notable example, where perturbative $\alpha$-corrections preserve the no-scale structure [28]. More precisely these are induced by the leading order $\alpha$-corrections to the $11 \mathrm{~d}$ low-energy effective action of M-theory after compactification on a Calabi-Yau fourfold. Furthermore, in the correct Kähler coordinates the Kähler potential has a homogeneity property. This is not immediately visible in the chiral description, but can be more easily inferred from the dual description in terms of linear multiplets given in that reference.
} 
more conceptually. Some insight can be gained from the scale invariance of string amplitudes at tree level. As argued in [4] for the heterotic string, this scale invariance induces a scaling property of the $10 \mathrm{D}$ effective Lagrangian. This in turn implies that the $4 \mathrm{D}$ effective Lagrangian obtained after compactification transforms with a certain weight under dilatations. However, in this case the only fields in the 4D theory that transform nontrivially are the dilaton and the space-time metric. In this way the scaling property of the Lagrangian does not constrain the form of the Kähler potential of the Kähler moduli. On the other hand for orientifold compactifications of the type II string we expect that the 4D fields will transform with different weights and so in principle the scaling property of the Lagrangian can lead to conditions upon the Kähler potential of the Kähler moduli. Recall that logarithmic Kähler potentials of homogeneous type possess an isometry associated with dilatations, where the respective Killing vector is given in eq. (4.25). It is conceivable that this isometry is induced by the scaling behaviour of the Lagrangian. Moreover, the scale-invariance of string amplitudes is explicitly broken at the quantum level and, hence, does not survive $g_{s}$-corrections [4]. This fact is in agreement with the observation that the $\alpha^{\prime}$-corrected Kähler potential in eq. (5.6) is still given by a logarithm of a homogeneous function while the $g_{s}$-corrected Kähler potential in eq. (5.8) is not.

One might go even further and ask whether the scaling behaviour is responsible for the weak no-scale property. However, the following evidence suggests otherwise. Consider, for example, that fluxes are absent so that the superpotential in the effective $4 \mathrm{D}$ theory vanishes identically. In this case only the scaling behavior of the kinetic terms constrains the form of the Kähler potential. Given that the fields in the spectrum transform with certain weights under dilatations one finds that a Kähler potential which is a homogeneous function of an appropriate degree will support the required weight of the kinetic terms. However, no such Kähler potential is of the weak no-scale type, which can be checked explicitly.

In conclusion, it is evident that the scaling behaviour of the Lagrangian implies the existence of the Killing vector in eq. (4.25), but is not strong enough to enforce the weak no-scale property and, hence, to uniquely single out logarithmic Kähler potentials of homogeneous type. Seen from this perspective, it could be possible that the weak no-scale property holds also at the quantum level, although our knowledge about the effective 4D action is far too incomplete to verify this yet. Moreover, since the scaling symmetry is broken at loop-order, any Kähler potential that supports the weak no-scale property beyond tree-level is very likely going to be of a non-homogeneous type.

Furthermore, it would be interesting to explore whether stringy $4 \mathrm{D}$ effective Lagrangians exist, which do not inherit a scaling property from string amplitudes and, thus, may constitute examples of non-homogeneous no-scale models. One may for instance wonder whether such models could arise from non-geometric string compactifications.

\section{Acknowledgments}

We would like to thank Ido Ben-Dayan, Jan Louis and Severin Lüst for important comments and discussions. We also thank Peter-Simon Dieterich, Klaus Fredenhagen, Constantin Muranaka and Alexander Westphal. This work was supported by the German 
Science Foundation (DFG) under the Collaborative Research Center (SFB) 676 Particles, Strings and the Early Universe, and by the Impuls und Vernetzungsfond of the Helmholtz Association of German Research Centers under grant HZ-NG-603.

\section{A Equivalence to the homogeneous Monge-Ampère equation}

In this appendix we explicitly demonstrate the equivalence between the no-scale condition for chiral fields, as given in eq. (2.7) and the HCMA, see eq. (3.1). Moreover, this demonstration will provide additional insight into the geometric meaning of the no-scale condition. Firstly, the HMCA, given in eq. (3.1), is equivalent to the statement that $Y_{T_{i} \bar{T}_{\bar{\jmath}}}$ has an eigenvalue zero. For $G$ given in eq. (3.2) we will now show that the respective eigenvector is given by

$$
G^{T_{i}} \equiv G^{T_{i} \bar{T}_{\bar{\jmath}}} G_{\bar{T}_{\bar{\jmath}}}
$$

Using eq. (3.2) we find that

$$
Y_{T_{i} \bar{T}_{\bar{\jmath}}}=\frac{Y}{p}\left(-G_{T_{i} \bar{T}_{\bar{\jmath}}}+\frac{1}{p} G_{T_{i}} G_{\bar{T}_{\bar{\jmath}}}\right) .
$$

Thus, we can compute

$$
Y_{T_{i} \bar{T}_{\bar{\jmath}}} G^{T_{i}}=\frac{Y}{p^{2}} G_{\bar{T}_{\bar{\jmath}}}\left(G^{T_{i}} G_{T_{i}}-p\right) .
$$

From the above equation we read off that

$$
Y_{T_{i} \bar{T}_{\bar{\jmath}}} G^{T_{i}}=0, \quad \text { iff } \quad G^{T_{i}} G_{T_{i}}=p
$$

which is nothing but the no-scale condition as defined in eq. (2.7). This completes the proof of the equivalence between solutions of the no-scale differential equation and the HCMA.

\section{B Duality transformation and matching the solutions}

\section{B.1 Duality transformation}

Let us discuss the duality transformation, which relates eq. (2.11) to a dual theory of chiral multiplets. We only reproduce the important formulae here, following [9, 12]. Let us introduce the Lagrangian

$$
\mathcal{L}=-3 \int E\left(F\left(L^{i}\right)+\frac{2}{3} L^{i}\left(T_{i}+\bar{T}_{i}\right)\right)+\frac{1}{2} \int \frac{E}{R} \mathrm{e}^{K / 2} W+\frac{1}{2} \int \frac{E}{\bar{R}} \mathrm{e}^{K / 2} \bar{W},
$$

where $L^{i}$ are only required to be real superfields. ${ }^{28}$ Moreover, $K\left(L^{i}\right)$ denotes the Kähler potential and $W$ the constant superpotential. On one hand the equations of motion for $T_{i}$ derived from eq. (B.1) are equivalent to eq. (2.10) and, hence, upon inserting the solution

\footnotetext{
${ }^{28}$ Whenever we discuss models with a shift-symmetry we will drop the bars on the indices for the complex conjugate scalars.
} 
we retain the theory in eq. (2.11) [9]. On the other hand, varying eq. (B.1) with respect to $L^{i}$ one finds $[9,12]$

$$
\frac{2}{3}\left(T_{i}+\bar{T}_{i}\right)+F_{L^{i}}-\frac{1}{3} K_{L^{i}}\left(F+\frac{2}{3} L^{j}\left(T_{j}+\bar{T}_{j}\right)\right)=0 .
$$

The above equation should be read as implicitly defining $L^{i}(T+\bar{T})$. The chiral theory is obtained after inserting $L^{i}(T+\bar{T})$ back into eq. (B.1). Since $L^{i}(T+\bar{T})$ is invariant under shifts $T_{i}-\bar{T}_{i} \rightarrow T_{i}-\bar{T}_{i}+C_{i}$ for some constants $C_{i}$, the dual chiral theory exhibits the lookedfor Peccei-Quinn shift-symmetry. Moreover, the Kähler potential of the chiral theory is given by $K$ where again we replace $L^{i}$ with the solution of eq. (B.2). The Einstein-frame condition in eq. (2.12) can now be recast into the form

$$
F\left(L^{j}\right)+\frac{2}{3} L^{i}\left(T_{i}+\bar{T}_{i}\right)=1 .
$$

Up to a constant factor, which can be removed via a Kähler transformation, we can rewrite eq. (2.13) as

$$
K(T+\bar{T})=\tilde{K}(L(T+\bar{T}))-2\left(T_{i}+\bar{T}_{i}\right) L^{i} .
$$

This equation identifies $K$ to be the Legendre transform of $\tilde{K}$. Furthermore, the kinetic terms of $L^{i}$ in eq. (2.14) yield kinetic terms for the real parts of $T_{i}$ in the dual chiral theory. The kinetic terms for the imaginary component of $T_{i}$ are obtained by dualizing the three-form $H^{i}$ to $d \operatorname{Im}\left(T_{i}\right)$ by means of Hodge duality. Performing the explicit procedure yields the exact form of the component Lagrangian eq. (2.3), where the Kähler metric is derived from $K$, see $[9,12]$.

Since we are interested in the scalar potential, we explicitly demonstrate the duality for $V$ given in eq. (2.15). For brevity let us introduce the following notation for the real components of the chiral scalars

$$
\phi_{i}=\frac{1}{2}\left(T_{i}+\bar{T}_{i}\right)
$$

Taking the derivative of eq. (B.3) with respect to $L^{i}$ and inserting eq. (B.2) shows that

$$
K_{L^{i}}=-4 L^{j} \frac{\partial \phi_{j}}{\partial L^{i}}
$$

Multiplying this equation with the inverse matrix of derivatives we read off

$$
L^{i}=-\frac{1}{4} K_{\phi_{i}} .
$$

Taking derivatives with respect to $\phi_{i}$ we find

$$
K_{\phi_{i} \phi_{j}}=-4 \frac{\partial L^{i}}{\partial \phi_{j}} .
$$

Using eq. (B.7) and eq. (B.8) we infer

$$
L^{i} K_{L^{i}}=L^{i} K_{\phi_{j}} \frac{\partial \phi_{j}}{\partial L^{i}}=K_{\phi_{i}} K^{\phi_{i} \phi_{j}} K_{\phi_{j}}=K_{T_{i}} K^{T_{i} \bar{T}_{j}} K_{\bar{T}_{j}},
$$

which shows that we obtain the correct scalar potential for a chiral theory with shiftsymmetric Kähler potential. 


\section{B.2 Matching the solutions upon dualization}

Using the general prescription for the duality transformation, we now show that the solution given in eqs. (3.27) and (3.28) matches the solution in eq. (3.19) after dualization to chiral multiplets. ${ }^{29}$ First, replacing the Kähler potential via eq. (3.23) in eq. (B.6) yields

$$
Y_{L^{i}}=\frac{4}{p} Y L^{j} \frac{\partial \phi_{j}}{\partial L^{i}}
$$

Using this equation we find that

$$
\phi_{i} Y_{\phi_{i}}=\phi_{i} Y_{L^{j}} \frac{\partial L^{j}}{\partial \phi_{i}}=\frac{4}{p} \phi_{i} L^{i} Y .
$$

Inserting eq. (B.3) into the above we obtain

$$
\phi_{i} Y_{\phi_{i}}=\frac{3}{p}(1-F) Y .
$$

Finally, using eqs. (3.27) and (3.28) we conclude that

$$
Y=\phi_{i} Y_{\phi_{i}}+\frac{3}{p} Y\left(\frac{L^{2}}{L^{1}}, \ldots, \frac{L^{n}}{L^{1}}\right) F_{(1)}\left(\frac{L^{2}}{L^{1}}, \ldots, \frac{L^{n}}{L^{1}}\right),
$$

where we have to read the equation as depending on the dual chiral coordinates. Comparing with eq. (3.19) and recalling the definition of the real variables in eq. (3.4) we find that the solutions match, after identifying

$$
\tilde{Y}\left(\frac{L^{2}}{L^{1}}, \ldots, \frac{L^{n}}{L^{1}}\right)=\frac{3}{p} Y\left(\frac{L^{2}}{L^{1}}, \ldots, \frac{L^{n}}{L^{1}}\right) F_{(1)}\left(\frac{L^{2}}{L^{1}}, \ldots, \frac{L^{n}}{L^{1}}\right),
$$

and the $n-1$ coordinates $u_{\alpha}$ are related to the real scalars inside the linear multiplets via

$$
u_{\alpha}=\frac{L^{\alpha}}{L^{1}}, \quad \alpha=1, \ldots, n-1,
$$

which again has to be read as an equality of functions each depending on $\left(T_{i}+\bar{T}_{i}\right)$. It remains to be seen that eq. (3.13) holds, i.e. we have to see whether $Y_{T_{i}}$ can be expressed as a function of the variables $u_{\alpha}$. Replacing $K$ via eq. (3.23) in eq. (B.7) we observe

$$
Y_{\phi_{i}}=\frac{4}{p} Y L^{i}
$$

Since $Y$ is homogeneous of degree -1 in the $L^{i}$, we can express the above as

$$
Y_{\phi_{i}}=\frac{4}{p} \frac{L^{i}}{L^{1}} Y\left(\frac{L^{2}}{L^{1}}, \ldots, \frac{L^{n}}{L^{1}}\right) \equiv g^{i}\left(\frac{L^{2}}{L^{1}}, \ldots, \frac{L^{n}}{L^{1}}\right),
$$

which is indeed a function of the variables $u_{\alpha}$ according to eq. (B.15).

\footnotetext{
${ }^{29}$ It is worth pointing out that theories of chiral and linear multiplets are in general dual only at the classical level. The reason for this is that the linear multiplet does not include an auxiliary field and that off-shell higher-derivative corrections for chiral multiplets exist, which in particular correct the scalar potential by powers of the chiral auxiliary. For the special case of string-derived effective actions these corrections were recently discussed in [29]. Furthermore, the no-scale condition in these higher-derivative supergravities was studied in [30].
} 
Let us finish this section by checking that the constraint equations in eq. (3.18) are automatically satisfied upon dualization. More precisely this amounts to the vanishing of the following object

$$
C^{\alpha}(\phi)=\phi_{i} \partial_{u_{\alpha}} g^{i}(u)+\partial_{u_{\alpha}} \tilde{Y}(u),
$$

where the $u_{\alpha}$ are understood as functions of the dual chiral fields $\phi_{i}$. To check that $C^{\alpha}$ vanish we must replace $\tilde{Y}\left(u_{\alpha}\right)$ and $g^{i}\left(u_{\alpha}\right)$ via eqs. (B.14) and (B.17) in eq. (B.18). Before performing this replacement we rewrite $\tilde{Y}$ and $g^{i}$ as functions of $L^{i}{ }^{30}$ Using eq. (3.27) for $Y(L)$ and similarly that $F_{(1)}(L)$ is a homogeneous function of degree one, we obtain

$$
\tilde{Y}\left(u_{\alpha}\right)=\frac{3}{p} Y(L) F_{(1)}(L) \quad \text { and } \quad g^{i}\left(u_{\alpha}\right)=\frac{4}{p} L^{i} Y(L) .
$$

After replacing these in eq. (B.18) and using the chain rule for derivatives we find

$$
C^{\alpha}(\phi)=\left(\frac{4}{p} \phi_{i} Y(L)+\frac{4}{p} \phi_{j} L^{j} Y_{L^{i}}(L)+\frac{3}{p} Y_{L^{i}}(L) F_{(1)}(L)+\frac{3}{p} Y(L) F_{(1) L^{i}}(L)\right) \frac{\partial L^{i}}{\partial u_{\alpha}} .
$$

Furthermore, using eqs. (3.28) and (B.3) the above reduces to

$$
C^{\alpha}(\phi)=\left(\frac{4}{p} \phi_{i} Y(L)+Y_{L^{i}}(L)+\frac{3}{p} Y(L) F_{(1) L^{i}}(L)\right) \frac{\partial L^{i}}{\partial u_{\alpha}} .
$$

Finally, by means of eqs. (B.2), (B.3) and (3.23) we directly obtain that the terms inside the parenthesis cancel. Thus, as promised $C^{\alpha}$ vanish.

Open Access. This article is distributed under the terms of the Creative Commons Attribution License (CC-BY 4.0), which permits any use, distribution and reproduction in any medium, provided the original author(s) and source are credited.

\section{References}

[1] E. Cremmer, S. Ferrara, C. Kounnas and D.V. Nanopoulos, Naturally vanishing cosmological constant in $N=1$ supergravity, Phys. Lett. B 133 (1983) 61 [INSPIRE].

[2] R. Barbieri, E. Cremmer and S. Ferrara, Flat and positive potentials in $N=1$ supergravity, Phys. Lett. B 163 (1985) 143 [INSPIRE].

[3] J.R. Ellis, A.B. Lahanas, D.V. Nanopoulos and K. Tamvakis, No-scale supersymmetric Standard Model, Phys. Lett. B 134 (1984) 429 [INSPIRE].

[4] E. Witten, Dimensional reduction of superstring models, Phys. Lett. B 155 (1985) 151 [INSPIRE].

[5] G. Dall'Agata and F. Zwirner, New class of $N=1$ no-scale supergravity models, Phys. Rev. Lett. 111 (2013) 251601 [arXiv:1308.5685] [INSPIRE].

[6] T.W. Chaundy, The differential calculus, Clarendon Press, Oxford U.K. (1935).

[7] D.B. Fairlie and A.N. Leznov, General solutions of the Monge-Ampère equation in n-dimensional space, J. Geom. Phys. 16 (1995) 385 [hep-th/9403134] [INSPIRE].

\footnotetext{
${ }^{30}$ From now on we omit writing the dependence upon the $\phi_{i}$ but it is implied.
} 
[8] J.-P. Derendinger, F. Quevedo and M. Quirós, The linear multiplet and quantum four-dimensional string effective actions, Nucl. Phys. B 428 (1994) 282 [hep-th/9402007] [INSPIRE].

[9] P. Binetruy, G. Girardi and R. Grimm, Supergravity couplings: a geometric formulation, Phys. Rept. 343 (2001) 255 [hep-th/0005225] [INSPIRE].

[10] T.W. Grimm and J. Louis, The effective action of $N=1$ Calabi-Yau orientifolds, Nucl. Phys. B 699 (2004) 387 [hep-th/0403067] [INSPIRE].

[11] S. Ferrara, C. Kounnas and F. Zwirner, Mass formulae and natural hierarchy in string effective supergravities, Nucl. Phys. B 429 (1994) 589 [Erratum ibid. B 433 (1995) 255] [hep-th/9405188] [INSPIRE].

[12] T.W. Grimm, The effective action of type-II Calabi-Yau orientifolds, Fortsch. Phys. 53 (2005) 1179 [hep-th/0507153] [INSPIRE].

[13] D. Fairlie, Comments on Galileons, J. Phys. A 44 (2011) 305201 [arXiv:1102.1594] [INSPIRE].

[14] S. Deser and J. Franklin, Symmetrically reduced Galileon equations and solutions, Phys. Rev. D 86 (2012) 047701 [arXiv:1206.3217] [INSPIRE].

[15] S.B. Giddings, S. Kachru and J. Polchinski, Hierarchies from fluxes in string compactifications, Phys. Rev. D 66 (2002) 106006 [hep-th/0105097] [INSPIRE].

[16] K. Becker, M. Becker, M. Haack and J. Louis, Supersymmetry breaking and $\alpha^{\prime}$ corrections to flux induced potentials, JHEP 06 (2002) 060 [hep-th/0204254] [INSPIRE].

[17] L. Covi, M. Gomez-Reino, C. Gross, J. Louis, G.A. Palma and C.A. Scrucca, De Sitter vacua in no-scale supergravities and Calabi-Yau string models, JHEP 06 (2008) 057 [arXiv: 0804.1073] [INSPIRE].

[18] A. Strominger, Yukawa couplings in superstring compactification, Phys. Rev. Lett. 55 (1985) 2547 [INSPIRE].

[19] L.J. Dixon, V. Kaplunovsky and J. Louis, On effective field theories describing $(2,2)$ vacua of the heterotic string, Nucl. Phys. B 329 (1990) 27 [INSPIRE].

[20] P. Candelas and X. de la Ossa, Moduli space of Calabi-Yau manifolds, Nucl. Phys. B 355 (1991) 455 [inSPIRE].

[21] M. Haack and J. Louis, $M$ theory compactified on Calabi-Yau fourfolds with background flux, Phys. Lett. B 507 (2001) 296 [hep-th/0103068] [INSPIRE].

[22] T.W. Grimm and J. Louis, The effective action of type IIA Calabi-Yau orientifolds, Nucl. Phys. B 718 (2005) 153 [hep-th/0412277] [INSPIRE].

[23] S. Gurrieri, J. Louis, A. Micu and D. Waldram, Mirror symmetry in generalized Calabi-Yau compactifications, Nucl. Phys. B 654 (2003) 61 [hep-th/0211102] [INSPIRE].

[24] M. Graña, J. Louis and D. Waldram, Hitchin functionals in $N=2$ supergravity, JHEP 01 (2006) 008 [hep-th/0505264] [INSPIRE].

[25] I. Benmachiche and T.W. Grimm, Generalized $N=1$ orientifold compactifications and the Hitchin functionals, Nucl. Phys. B 748 (2006) 200 [hep-th/0602241] [INSPIRE].

[26] M. Graña, J. Louis and D. Waldram, $\mathrm{SU}(3) \times \mathrm{SU}(3)$ compactification and mirror duals of magnetic fluxes, JHEP 04 (2007) 101 [hep-th/0612237] [INSPIRE]. 
[27] I. Antoniadis, S. Ferrara, R. Minasian and K.S. Narain, $R^{4}$ couplings in $M$ and type-II theories on Calabi-Yau spaces, Nucl. Phys. B 507 (1997) 571 [hep-th/9707013] [INSPIRE].

[28] T.W. Grimm, J. Keitel, R. Savelli and M. Weissenbacher, From M-theory higher curvature terms to $\alpha^{\prime}$ corrections in F-theory, arXiv:1312.1376 [INSPIRE].

[29] D. Ciupke, J. Louis and A. Westphal, Higher-derivative supergravity and moduli stabilization, JHEP 10 (2015) 094 [arXiv: 1505.03092] [INSPIRE].

[30] S. Cecotti, S. Ferrara and L. Girardello, Flat potentials in higher derivative supergravity, Phys. Lett. B 187 (1987) 327 [INSPIRE]. 